\title{
Adaptive Fractional-Order Nonsingular Fast Terminal Sliding Mode Control for Manipulators
}

\author{
Xin Zhang ${ }^{1,2}$ and Ran Shi $\mathbb{D}^{1}$ \\ ${ }^{1}$ School of Automation \& Electrical Engineering of Lanzhou Jiaotong University, Lanzhou 730070, China \\ ${ }^{2}$ Gansu Provincial Engineering Research Center for Artificial Intelligence and Graphics \& Image Processing, \\ Lanzhou 730070, China \\ Correspondence should be addressed to Ran Shi; 12201420@stu.lzjtu.edu.cn
}

Received 3 August 2021; Accepted 6 September 2021; Published 21 September 2021

Academic Editor: Xianggui Guo

Copyright (C) 2021 Xin Zhang and Ran Shi. This is an open access article distributed under the Creative Commons Attribution License, which permits unrestricted use, distribution, and reproduction in any medium, provided the original work is properly cited.

\begin{abstract}
When the manipulator system is subject to unknown disturbance, in order to improve the tracking accuracy of the manipulator, this paper designs a fractional-order nonsingular fast terminal sliding mode (FONFTSM) controller. The controller is divided into three parts. First of all, in order to improve the performance of the sliding stage, this paper designs a FONFTSM surface. By introducing a fractional-order operator, the convergence speed and accuracy of the system state are effectively improved. Secondly, in view of the problems of large chattering and slow convergence speed in the reaching stage, this paper designs a variable exponential power-reaching law (VEPRL), which has the ability to change the exponential coefficients according to the system state adaptively. At the same time, an adaptive law is designed to adjust the coefficients of the reaching law adaptively, which enhances the robustness of the control system. Finally, a disturbance observer is used to estimate the unknown external disturbance in real time so as to perform feedforward compensation for the control system, which effectively improves the accuracy of the manipulator control system. The stability of the manipulator control system is proved by the Lyapunov function. Simultaneously, the controller designed in this paper is compared with different controllers, which proves that the controller designed in this paper has strong robustness, high control accuracy, and fast convergence speed.
\end{abstract}

\section{Introduction}

In recent years, manipulators have been widely used in industrial fields [1-4]. The use of manipulators can help people complete complex and repetitive tasks. For manipulators, most tasks require precise tracking accuracy. However, since the manipulator itself is a nonlinear system with strong coupling and parameter uncertainty, it is difficult for traditional PID controllers to achieve better tracking accuracy and speed in the presence of external disturbance. Therefore, for nonlinear systems, scholars have proposed many control methods [5], time delay control method [6-10], sliding mode control (SMC) method [11-15], fuzzy control method [16, 17], and backstepping control method [18]. Among them, the SMC method is a nonlinear control method. Its control method is simple, which is suitable for the control of nonlinear systems. At the same time, SMC is robust and insensitive to unknown disturbances and is suitable for applications in complex environments.

SMC is divided into the sliding stage and the reaching stage. For the sliding stage, the traditional SMC adopts a linear sliding mode surface, and the system state can only gradually converge to the equilibrium point, and the convergence time tends to infinity. Although it is possible to speed up the system state convergence speed by adjusting the parameters of the sliding mode surface, this can only be done at the cost of larger control input and is not used in actual engineering. Another disadvantage of the linear sliding mode surface is the chattering problem. Tang [19] proposed a terminal sliding mode surface. By designing a nonlinear sliding mode surface, a finite-time convergence state was 
achieved. Compared with the linear sliding mode surface, the convergence speed when the system state is close to the equilibrium point is accelerated, and the control input is in a proper range at the same time. However, the terminal sliding mode surface has the problem of singularity. When it is close to some points of the equilibrium point, the control input will tend to be infinite, which is not conducive to use in practical engineering. Yang and Yang [20] designed a fast terminal sliding mode surface. Although the speed of the system state converging to the equilibrium point has been further improved, it has the same problem of singularity as the terminal sliding mode surface. Feng et al. [21] designed a nonsingular terminal sliding mode surface, which effectively solved the singularity problem while maintaining the finitetime convergence characteristics of the system state. Tian et al. [22] designed a new type of nonsingular fast terminal sliding mode surface. By introducing a saturation function into the controller, the singularity problem of the traditional fast terminal sliding mode surface is effectively solved. The above sliding mode surfaces are integer-order sliding mode surfaces, and fractional-order sliding mode surfaces are not involved. The fractional-order calculus operator is a further extension of the integer order, and the fractional-order controller has better flexibility and tracking accuracy. Jie et al. [23] designed a new type of fractional-order terminal sliding mode surface, and at the same time, the unknown disturbance was estimated in real time through the disturbance observer. The experimental results proved that the controller has good tracking accuracy and strong robustness. Duc et al. [24] introduced a fractional-order differential operator on the basis of the traditional nonsingular terminal sliding mode surface and, at the same time, adaptively adjusted the switching coefficients in the SMC through fuzzy control. The simulation results show that compared with the integer-order controller, the fractional-order controller has better tracking accuracy. Although the above sliding mode surface introduces a fractional-order operator, the speed of the system state convergence can be further improved.

The chattering problem inherent in SMC is also one of the hot research issues. Reaching law method [25, 26], saturation function method [27], and high-order sliding mode method [28-30] can effectively solve the chattering problem in SMC. The reaching law method reduces the chattering in SMC, and at the same time, it effectively improves the dynamic performance of the reaching stage. Gao and Hung [31] proposed the traditional exponential reaching law and the power-reaching law, but the abovementioned reaching laws all have shortcomings. Under the action of the exponential reaching law, although the system state converges fast, there is a large amount of chattering. Although the power-reaching law is close to the sliding mode surface, the chattering amplitude is small, but the system state convergence speed is too slow. Li and Cai [32] proposed the double-power-reaching law. Under the action of the double-power-reaching law, the system state has a faster convergence rate in the overall process of reaching the sliding mode surface. Pan et al. [33] designed a new type of reaching law on the basis of the double-power-reaching law. However, there are many parameters of the reaching law, and it is more complicated to implement in actual situations. Yang and Chen [34] designed a multipower-reaching law. Although the exponential coefficients can be adjusted adaptively according to the system state, the reaching law coefficients do not have the ability to adapt. Most of the abovementioned reaching laws do not have the ability to adapt, and the coefficients of the reaching law cannot be adjusted adaptively according to the system state.

The unknown external disturbance is difficult to obtain directly in actual engineering. It is often necessary to set the value of the switching item to be too large to ensure the stability of the control system, but this will lead to the emergence of chattering problems. Li et al. [35] aimed at the problem of permanent magnet linear motor position control, using a finite-time disturbance observer to estimate the external disturbance and use this estimated value as the compensation of the control system. In [36], real-time disturbance estimation is carried out for the photovoltaic grid-connected inverter by the disturbance observer, which greatly reduces chattering. Simulation results verify the effectiveness of the control method. The introduction of the disturbance observer can estimate the unknown disturbance and then perform feedforward compensation for the control system.

To sum up, compared with the deficiencies in the above literature, an adaptive FONFTSM controller is proposed in this paper to solve the trajectory tracking control problem of the manipulator. Specific contributions are as follows:

(1) In this paper, a FONFTSM is designed, which effectively improves the speed and accuracy of the system state converging to the equilibrium point. At the same time, the coefficients of the reaching law are adjusted through the adaptive law to further improve the robustness of the system.

(2) A disturbance observer is introduced to estimate the unknown external disturbance in real time and then make feedforward compensation, which improves the tracking accuracy of the manipulator control system.

(3) The stability of the manipulator control system is proved by the Lyapunov function. At the same time, the superiority of the controller designed in this paper is verified by simulation.

The rest of the arrangement is as follows. Section 2 introduces some basic knowledge. Section 3 introduces the sliding mode controller designed in this paper. In order to show the superiority of the controller designed in this paper, Section 4 carries on the simulation verification, and Section 5 gives the conclusion of this paper.

\section{Basic Knowledge}

\subsection{Some Basic Definitions and Lemmas}

Definition 1. Functions which commonly used Caputo fractional-order derivatives are defined as follows [37]: 


$$
{ }_{t_{0}} D_{t}^{\alpha} f(t)= \begin{cases}\frac{\int_{t_{0}}^{t}\left(f^{m}(\tau) /(t-\tau)^{a-m+1}\right) \mathrm{d} \tau}{\Gamma(m-\alpha)}, & m-1<\alpha<m, \\ \frac{\mathrm{d}^{m}}{\mathrm{~d} t^{m}} f(t), & \alpha=m,\end{cases}
$$

where $\alpha$ represents the order of fractional order, $m$ is the first integer greater than order $\alpha$, and $\Gamma(\cdot)$ represents the gamma function.

Lemma 1. The fractional-order equation is expressed as follows [38]:

$$
t_{0} D_{t}^{\alpha} f(t)=x(f(t)),(0<\alpha<1, f(t) \in \mathbb{R}) .
$$

When the following inequality is satisfied,

$$
f(t) x(f(t))<0, \forall f \neq 0 .
$$

Then, $f(t)=0$ is the equilibrium point of the system corresponding to equation (2), and the system corresponding to equation (2) is asymptotically stable. AguilaCamacho et al. [38] give the concrete proof process.

Lemma 2. For calculus operators $I_{c+}^{b}$ and $I_{d-}^{b}$, where powers $b$ and $R(b)$ are bounded at $L_{p}(a, b), 1<p \leq \infty$, the following expression exists [37]:

$$
\begin{array}{r}
\left\|I_{c+}^{b}\right\|_{p} \leq H\|y\|_{p}, \\
\left\|I_{d-}^{b}\right\|_{p} \leq H\|y\|_{p}, \\
\left(H=\frac{(d-c)^{\Re(b)}}{\Re(b)|\Gamma(b)|}\right) .
\end{array}
$$

For the proof of Lemma 2, we refer to [37].

Lemma 3. There is a nonlinear system expressed as $\dot{x}=f(x, u)$, and there is a functional relationship expressed as follows [39]:

$$
\dot{V}(x) \leq-\mu V^{\delta}(x)+\gamma,
$$

where $0<\gamma<\infty, \mu>0,0<\delta<1$, and $V(x)$ is a continuous function. Then, the nonlinear system $\dot{x}=f(x, u)$ is called a finite-time stable system. At the same time, the system state can converge to the corresponding region in finite time:

$$
\lim _{\theta \rightarrow \theta_{0}} x \in\left(V^{\delta}(x) \leq \frac{\gamma}{(1-\theta) \mu}\right),
$$

where $0<\theta_{0}<1$. At the same time, the time taken for the system state to reach the corresponding area is expressed as follows:

$$
T \leq \frac{V^{1-\delta}\left(x_{0}\right)}{\left[\mu \theta_{0}(1-\delta)\right]},
$$

where $V\left(x_{0}\right)$ represents the initial value of $V(x)$. For the specific proof process, we refer to [39].

In order to express more succinct, this paper uses the fractional-order symbol $D^{\alpha}$ instead of the Caputo fractional symbol $t_{0} D_{t}^{\alpha}$.

2.2. Dynamics Model of the Manipulator. According to the analysis of the Lagrange formula method [19], the dynamics model of the two-joint manipulator is shown as follows:

$$
M(q) \ddot{q}+C(q, \dot{q}) \dot{q}+G(q)=\tau+\tau_{d},
$$

where $q, \dot{q}, \ddot{q} \in R^{n}$ are the joint angle, joint angular velocity, and joint acceleration of the manipulator system, $M(q) \in R^{n \times n}$ is the nonsingular positive definite inertia matrix, $C(q, \dot{q}) \in R^{n \times n}$ is the centrifugal and Coriolis force matrix, $G(q)$ is the gravity vector,and $\tau_{d} \in R^{n}$ is the lumped uncertainty of the manipulator system. In order to make the actual trajectory accurately track the expected trajectory, certain assumptions need to be made, which are expressed as follows.

Assumption 1. Let $d=M^{-1} \tau_{d},|d| \leq D, M$ be reversible, and $D$ be a normal number.

Because the manipulator system is a system with high nonlinearity, strong coupling, and parameter uncertainty, in order to enable the actual trajectory of the manipulator to accurately track the desired trajectory, a suitable robust controller needs to be designed. This paper designs an adaptive FONFTSM controller, which effectively improves the trajectory tracking accuracy of the manipulator.

\section{Manipulator Control Scheme Design}

3.1. FONFTSM Controller Design. The traditional SMC uses linear sliding mode surface $s=\dot{e}+a_{1} e$ and $e=q-q_{d} \cdot q$ is the actual trajectory of the manipulator system and $q_{d}$ is the desired trajectory of the manipulator system. Under the action of the linear sliding mode surface, the system state does not have the finite-time convergence ability and can only gradually converge to the system equilibrium point. The terminal sliding mode surface solves the shortcomings of the linear sliding mode surface that the convergence speed is slow and the convergence time tends to infinity, but when the system state is close to the equilibrium point, singularity problems will occur at some points, and the control input will tend to infinity.

Yang and Yang [20] designed a fast terminal sliding mode surface, and the specific expression is as follows:

$$
s=\dot{e}+a_{1}|e|^{k_{1}} \operatorname{sign}(e)+a_{2}|e|^{k_{2}} \operatorname{sign}(e),
$$

where $a_{1}>0, a_{2}>0,0<k_{2}<1$, and $k_{1}>1$. It can be seen that the system state has a faster convergence speed when it is far from the equilibrium point or close to the equilibrium point, but the same as the terminal sliding mode surface; it will cause singularity problems. In order to solve the singular problem and improve the convergence rate of the system state, inspired by [22], this paper designs a FONFTSM surface, which is expressed as follows: 


$$
s=D^{\alpha} e+a_{1}|e|^{k_{1}} \operatorname{sign}(e)+a_{2}|e|^{k_{2}} \operatorname{sign}(e),
$$

where $a_{1}>0, a_{2}>0$, and $a_{1}$ and $a_{2}$ are expressed as follows:

$$
\begin{array}{ll}
k_{1}=\frac{o_{1}+1}{2}+\frac{o_{1}-1}{2} \operatorname{sign}(|e|-1), & o_{1}>1, \\
k_{2}=\frac{o_{2}+1}{2}+\frac{1-o_{2}}{2} \operatorname{sign}(|e|-1), & 0<o_{2}<1 .
\end{array}
$$

It can be seen from equation (10) that the exponential term has the ability to adapt and can accelerate the convergence to the equilibrium point according to the system state. In order to prove the stability of the sliding mode surface designed in this paper, it can be analyzed according to Lemma 1. When the system state reaches the sliding mode surface, $s=0$, at this time, equation (10) is

$$
D^{\alpha} e+a_{1}|e|^{k_{1}} \operatorname{sign}(e)+a_{2}|e|^{k_{2}} \operatorname{sign}(e)=0 .
$$

According to the analysis of Lemma 1, $-e\left(a_{1}|e|^{k_{1}} \operatorname{sign}(e)+a_{2}|e|^{k_{2}} \operatorname{sign}(e)\right)<0, \forall e \neq 0$. Therefore, it can be obtained that the sliding mode surface corresponding to equation (11) is asymptotically stable, and the system state can converge to the equilibrium point. The design of the abovementioned FONFTSM surface only improves the control performance of the manipulator in the sliding phase. How to improve the system state when it is in the reaching phase is also a hot research topic. This paper designs an adaptive VEPRL, which is expressed as follows:

$$
\dot{s}=-c_{1}|s|^{b} \operatorname{sign}(s)-c_{2} \operatorname{arsh}(s),
$$

where $b=\left\{\begin{array}{ll}b_{1}, & s \geq 1 \\ b_{2}, & s<1\end{array}\right.$ and $\widetilde{c}_{1}=\widehat{c}_{1}-c_{1}$. The values of $c_{1}$ and $c_{2}$ are both greater than 0 and $\operatorname{arsh}(\cdot)$ represents the inverse hyperbolic sine function. When $s \geq 1$, the coefficient of the exponential term is greater than 1, speeding up the speed of the system state approaching the sliding mode surface. When $s<1$, the coefficient of the exponential term is less than 1, speeding up the speed of the system state tending to the sliding mode surface. At the same time, an inverse hyperbolic sine function is introduced, which plays the role of smoothing and limiting. In order to further enhance the adaptive ability of the reaching law, this paper designs an adaptive law to adjust the coefficients of the reaching law in real-time. The specific expression is as follows:

$$
\begin{cases}\dot{\hat{c}}_{1}=p \operatorname{sign}\left(|s|-p_{1}\right), & \widehat{c}_{1} \leq c_{1 \max }, \\ \widehat{c}_{1}=c_{1 \max }, & \widehat{c}_{1} \geq c_{1 \max },\end{cases}
$$

where $p$ is a number greater than $0, p_{1}$ is a small positive number, and $c_{1 \max }$ is the maximum value of the reaching law coefficient. Combining equations (12) and (13), we can get the following equation:

$$
\dot{s}=-\widehat{c}_{1}|s|^{b} \operatorname{sign}(s)-c_{2} \operatorname{arsh}(s) .
$$

By combining formulae (14), (10), and (8), the control input of the manipulator system can be obtained as

$$
\begin{aligned}
\tau= & M^{1-\alpha}\left(-\widehat{c}_{1}|s|^{b} \operatorname{sign}(s)-c_{2} \operatorname{arsh}(s)-k_{1} a_{1}|e|^{k_{1}-1} \dot{e}-k_{2} a_{2}|e|^{k_{2}-1} \dot{e}\right)+C \dot{q}+G \\
& +M \ddot{q}_{d}-M c_{3} \operatorname{sign}(s)-M d,
\end{aligned}
$$

where $c_{3}>0$, and the magnitude of external disturbance $d$ is difficult to obtain in actual situations. It is usually necessary to suppress the disturbance by switching items to ensure the stability of the control system. Usually, the value of the switching item is larger. However, the switching item often contains a discontinuous function $\operatorname{sign}(\cdot)$, which causes a large-scale chattering phenomenon, resulting in a decrease in control accuracy and an increase in the amplitude of the chattering. This paper introduces a disturbance observer to estimate the time-varying disturbance from the outside world in real time, which effectively solves this problem. Inspired by [40], a disturbance observer is introduced, which is expressed as follows:

$$
\left\{\begin{array}{l}
\dot{z}_{1}=z_{2}+\lambda \nu_{1} \delta\left(\dot{q}-z_{1}, \kappa_{1}, \xi_{1}\right)+\mu \\
\dot{z}_{2}=\lambda^{2} v_{2} \delta\left(\dot{q}-z_{1}, \kappa_{2}, \xi_{2}\right)
\end{array}\right.
$$

where $v_{1}, v_{2}>0, \quad v_{1} \geq 2 \sqrt{\nu_{2}}$, and $\lambda \geq 0 . \quad \mu$ is specifically expressed as $M(q)^{-1}(\tau-C(q, \dot{q}) \dot{q}-G(q)), z_{1}$ and $z_{2}$ represent the estimated values of $\dot{q}$ and $d$, respectively, $\kappa$ has a value range of $\kappa \in(0.5,1)$, and $\xi$ has a value range of $\xi \in(1,1.5)$, specifically expressed in this paper as

$$
\delta(x, \gamma, \xi)= \begin{cases}|x|^{\kappa} \operatorname{sign}(x), & |x|<1, \\ |x|^{\xi} \operatorname{sign}(x), & |x| \geq 1 .\end{cases}
$$

For the specific proof process, refer to [40]; by introducing a disturbance observer to estimate the external disturbance in real-time, there is $|d-\widehat{d}|=\widetilde{d}<v$, where $v$ is a small integer. At the same time, it effectively solves the problems of large chattering amplitude and low tracking accuracy. At this time, the control input is

$$
\begin{aligned}
\tau= & \mathrm{MD}^{1-\alpha}\left(-\widehat{c}_{1}|s|^{b} \operatorname{sign}(s)-c_{2} \operatorname{arsh}(s)-k_{1} a_{1}|e|^{k_{1}-1} \dot{e}-k_{2} a_{2}|e|^{k_{2}-1} \dot{e}\right)+C \dot{q}+G \\
& +M \ddot{q}_{d}-M c_{3} \operatorname{sign}(s)-M \widehat{d} .
\end{aligned}
$$


Since $0<k_{2} \leq 1, k_{2} a_{2}|e|^{k_{2}-1} \dot{e}$ have singularity problems, influenced by [22]; saturation function is introduced in this paper to solve the singularity problems, which is specifically expressed as follows:

$$
\begin{aligned}
& \operatorname{sat}\left(k_{2} a_{2}|e|^{k_{2}-1} \dot{e}, h\right) \\
& =\left\{\begin{array}{l}
k_{2} a_{2}|e|^{k_{2}-1} \dot{e},\left.\quad\left|k_{2} a_{2}\right| e\right|^{k_{2}-1} \dot{e} \mid<h, \\
\left.h \operatorname{sign}\left(k_{2} a_{2}|e|^{k_{2}-1} \dot{e}\right) \quad\left|k_{2} a_{2}\right| e\right|^{k_{2}-1} \dot{e} \mid \geq h .
\end{array}\right.
\end{aligned}
$$

According to [22], equation (20) ignores the singularity region, when $k_{2} a_{2}|e|^{k_{2}-1} \dot{e}>h$. The system state does not stay in the singular region. Although the singular interval exists, the existence of the singular interval has little influence on the manipulator system and does not affect the stability of the control system [41]. In order to ensure the nonsingularity of the controller, the value of $h$ can be $h>k_{2} a_{2}|e|_{\max }^{k_{2}-1} \dot{e}_{\max }$. The control input is transformed to

$$
\begin{aligned}
\tau= & M^{1-\alpha}\left(-\widehat{c}_{1}|s|^{b} \operatorname{sign}(s)-c_{2} \operatorname{arsh}(s)-k_{1} a_{1}|e|^{k_{1}-1} \dot{e}-\operatorname{sat}\left(k_{2} a_{2}|e|^{k_{2}-1} \dot{e}, h\right)\right)+C \dot{q}+G \\
& +M \ddot{q}_{d}-M c_{3} \operatorname{sign}(s)-M \hat{d} .
\end{aligned}
$$

At this time, there is no singularity problem in the manipulator control system, which is effectively solved. Next, the stability of the controller designed in this paper is proved.

3.2. Stability Analysis. Substituting equation (21) into equation (8), the following equation can be obtained:

$$
\dot{s}=-\widehat{c}_{1}|s|^{b} \operatorname{sign}(s)-c_{2} \operatorname{arsh}(s)-D^{\alpha-1} c_{3} \operatorname{sign}(s)+D^{\alpha-1} \tilde{d} .
$$

Let the Lyapunov function be

$$
V_{1}=\frac{1}{2} s^{2}+\frac{1}{2} \widetilde{c}_{1}^{2}
$$

The derivative of equation (23) in the first order can be obtained:

$$
\dot{V}_{1}=s \dot{s}+\widetilde{c}_{1} \dot{\tilde{c}}_{1}
$$

Substitute equation (22) into equation (24) to obtain

$$
\begin{aligned}
\dot{V}_{1} & =s \dot{s}+\widetilde{c}_{1} \dot{\tilde{c}}_{1} \\
& =s\left(-\widehat{c}_{1}|s|^{b} \operatorname{sign}(s)-c_{2} \operatorname{arsh}(s)-D^{\alpha-1} c_{3} \operatorname{sign}(s)+D^{\alpha-1} \widetilde{d}\right)+\tilde{c}_{1} \dot{\bar{c}}_{1} .
\end{aligned}
$$

Since the error value $v$ of the disturbance observer is a very small value, the value of $c_{3}$ is selected to make $D^{\alpha-1}\left(-c_{3} \operatorname{sign}(s)+\widetilde{d}\right) \leq 0$, and formula (25) is transformed into

$$
\begin{aligned}
\dot{V}_{1} & =s \dot{s}+\widetilde{c}_{1} \dot{\tilde{c}}_{1} \\
& =s\left(-\widehat{c}_{1}|s|^{b} \operatorname{sign}(s)-c_{2} \operatorname{arsh}(s)\right)+\widetilde{c}_{1} \dot{\tilde{c}}_{1} .
\end{aligned}
$$

By transforming equation (26), we can obtain

$$
\begin{aligned}
\dot{V}_{1} & \leq s\left(-\widehat{c}_{1}|s|^{b} \operatorname{sign}(s)\right)+\widetilde{c}_{1} \dot{\tilde{c}}_{1} \\
& \leq-\widehat{c}_{1}|s|^{b+1}+\widetilde{c}_{1} \dot{\tilde{c}}_{1} .
\end{aligned}
$$

Further analysis of equation (27) can be obtained:

$$
\begin{aligned}
\dot{V}_{1} & \leq-\widehat{c}_{1}|s|^{b+1}+\tilde{c}_{1} \dot{\tilde{c}}_{1} \\
& \leq-\left(\widehat{c}_{1} 2^{(b+1) / 2}\left(\frac{1}{2} s^{2}\right)^{(b+1) / 2}+\left(\frac{1}{2} \widetilde{c}_{1}^{2}\right)^{(b+1) / 2}\right)+\left(\frac{1}{2} \widetilde{c}_{1}^{2}\right)^{(b+1) / 2}+\tilde{c}_{1} \dot{\tilde{c}}_{1} .
\end{aligned}
$$

Influenced by [29], let $\varphi_{0}=\min \left(\widehat{c}_{1} 2^{(b+1) / 2}, 1\right)$; combined with equation (28), we can obtain

$$
-\widehat{c}_{1} 2^{(b+1) / 2}\left(\frac{1}{2} s^{2}\right)^{(b+1) / 2}-\left(\frac{1}{2} \widetilde{c}_{1}^{2}\right)^{(b+1) / 2} \leq-\varphi_{0} V_{1}^{(b+1) / 2} .
$$

Combining with equation (28) and equation (29), we can get the following formula:

$$
\dot{V}_{1} \leq-\varphi_{0} V_{1}^{(b+1) / 2}+\left(\frac{1}{2} \widetilde{c}_{1}^{2}\right)^{(b+1) / 2}+\widetilde{c}_{1} \dot{\tilde{c}}_{1} .
$$

According to the adaptive law (14) designed in this paper, it can be concluded that $\left((1 / 2) c_{1}^{2}\right)^{(b+1) / 2}+\tilde{c}_{1} \dot{\tilde{c}}_{1}$ is bounded, and let $\Phi=\left((1 / 2) \widetilde{c}_{1}^{2}\right)^{(b+1) / 2}+\widetilde{c}_{1} \dot{\tilde{c}}_{1}$. When the range of $s$ is less than $1,0<b<1$ and $0<(b+1) / 2<1$. Therefore, according to Lemma 3 , the state of the system will converge to the following region in a finite time:

$$
\phi=|s| \leq 2^{(1 / 2)}\left(\frac{\emptyset}{(1-\theta) \varphi_{0}}\right)^{(1 /(b+1))} .
$$

To sum up, under the action of the controller designed in this paper, the system state can converge to the region $\phi$ represented by equation (32) in finite time. When the system state reaches $\phi$, the FONFTSM surface is expressed as

$$
\begin{aligned}
\dot{e} & +\left(a_{1}-\frac{1}{2} D^{1-\alpha} s\left[D^{1-\alpha}|e|^{k_{1}} \operatorname{sign}(e)\right]^{-1} D^{1-\alpha}|e|^{k_{1}} \operatorname{sign}(e)\right) \\
& +\left(a_{2}-\frac{1}{2} D^{1-\alpha} s\left[D^{1-\alpha}|e|^{k_{2}} \operatorname{sign}(e)\right]^{-1}\right) D^{1-\alpha}|e|^{k_{2}} \operatorname{sign}(e)=0 .
\end{aligned}
$$

When equation (33) is satisfied, equation (32) has the same form as equation (12), and the system state can converge to the equilibrium point: 

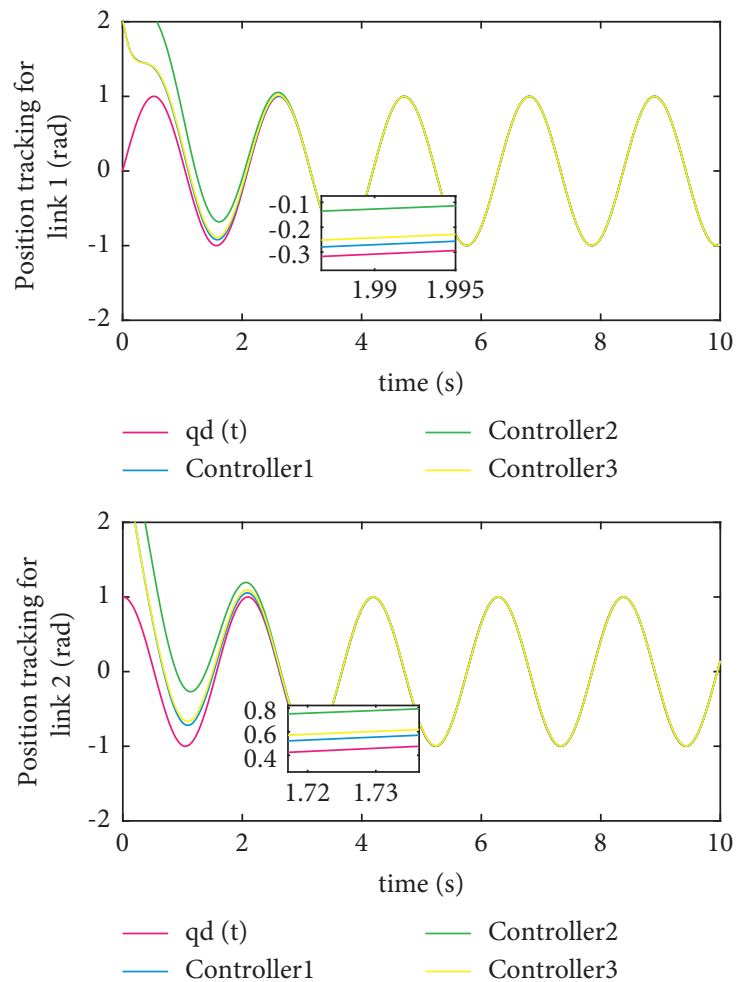

Figure 1: Comparison of manipulator trajectory tracking.
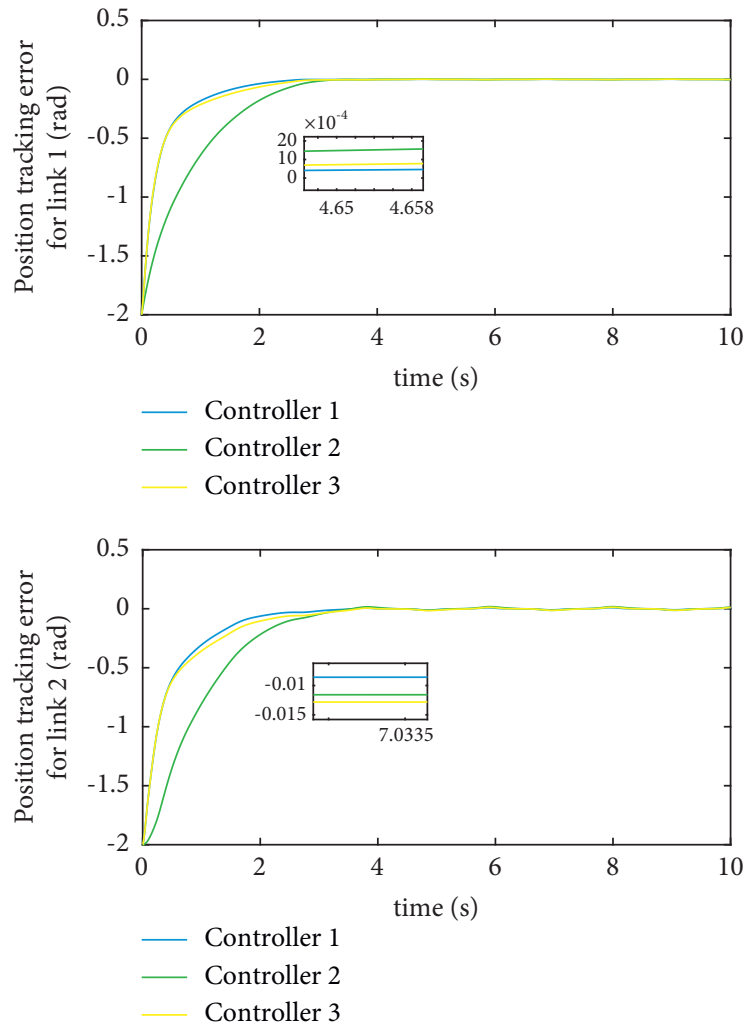

Figure 2: Comparison of tracking error of manipulator. 
TABLE 1: Comparison of RMSE (rad) of 3 controllers ( $0 \mathrm{~s})$.

\begin{tabular}{lcr}
\hline Controller & Joint 1 & Joint 2 \\
\hline Controller 1 & 0.2510 & 0.2883 \\
Controller 2 & 0.3884 & 0.4565 \\
Controller 3 & 0.2523 & 0.2900 \\
\hline
\end{tabular}

TABLE 2: Comparison of RMSE (rad) of 3 controllers ( $3 \mathrm{~s}$ ).

\begin{tabular}{llr}
\hline Controller & Joint 1 & Joint 2 \\
\hline Controller 1 & 0.0007 & 0.0005 \\
Controller 2 & 0.0022 & 0.0108 \\
Controller 3 & 0.0014 & 0.0009 \\
\hline
\end{tabular}
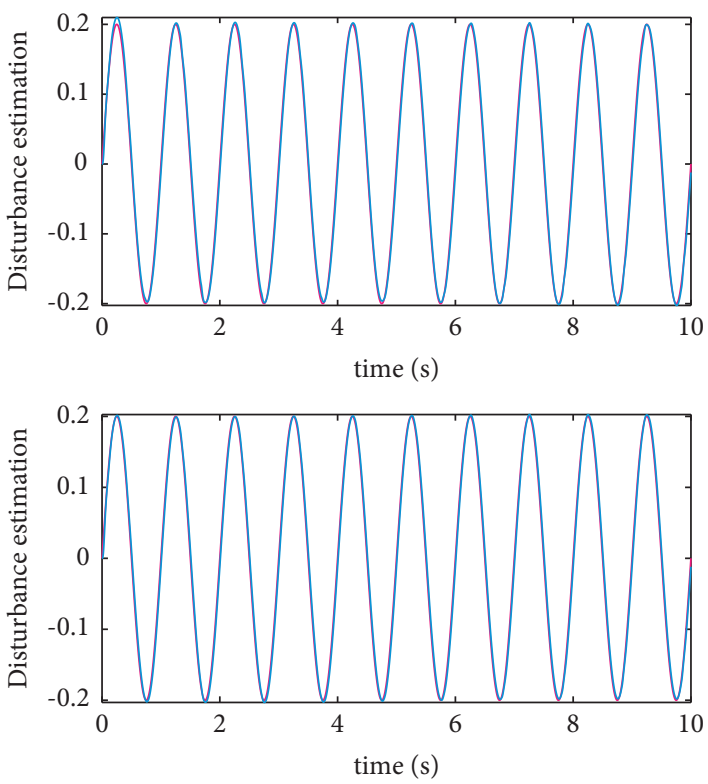

FIGURE 3: Disturbance estimation.

$$
\begin{aligned}
& a_{1}-\frac{1}{2} D^{1-\alpha} s\left[D^{1-\alpha}|e|^{k_{1}} \operatorname{sign}(e)\right]^{-1}>0, \\
& a_{2}-\frac{1}{2} D^{1-\alpha} s\left[D^{1-\alpha}|e|^{k_{2}} \operatorname{sign}(e)\right]^{-1}>0 .
\end{aligned}
$$

Assuming $a_{3}=2 \max \left(a_{1}, a_{2}\right)$ and $k_{3}=\max \left(k_{1}, k_{2}\right)$, we can obtain

$$
a_{3}-D^{1-\alpha} s\left[D^{1-\alpha}|e|^{k_{3}} \operatorname{sign}(e)\right]^{-1}>0 .
$$

According to Lemma 2 and equation (33), let $p \longrightarrow \infty$; we get the following inequality:

$$
\left.\left|D^{1-\alpha}\right| e\right|^{k_{3}} \operatorname{sign}(e) \mid \leq a_{3}^{-1} K_{1} \Delta .
$$

According to Lemma 3 let $p \longrightarrow \infty$; we can obtain

$$
\left.\left.\left|D^{1-\alpha}\right| e\right|^{k_{3}} \operatorname{sign}(e)\left|\leq K_{2}\right| e\right|_{\max } ^{k_{3}} \text {. }
$$

Set parameter $\omega \geq 1$; formula (35) becomes

$$
\left.\left.\left|D^{1-\alpha}\right| e\right|^{k_{3}} \operatorname{sign}(e)\left|=\omega^{-1} K_{2}\right| e\right|_{\max } ^{k_{3}} .
$$

Combining formulae (37) and (36), we can get the following inequality:

$$
\omega^{-1} K_{2}|e|_{\max }^{k_{3}} \leq a_{3}^{-1} K_{1} \Delta .
$$

The equation transformation of equation (37) can be obtained:

$$
|e| \leq|e|_{\max } \leq\left(a_{3}^{-1} K_{1} \Delta \omega K_{2}^{-1}\right)^{1 / k_{3}} .
$$

In summary, under the action of the controller designed in this paper, no matter whether the system state is in a sliding mode or a reaching mode, the system state can converge to the corresponding area. In the next step, in order to prove the superiority of the controller designed in this paper, compare and analyze the controllers with different references.

\section{Simulation Comparison Analysis}

In this section, three different controllers are simulated and verified to prove the superiority of the FONFTSM controller designed in this paper. First of all, this article uses a two-joint 
manipulator dynamic model, which is specifically expressed as follows:

$$
\begin{aligned}
M(q) & =\left[\begin{array}{cc}
x_{1}+x_{2}+2 x_{3} \cos \left(q_{2}\right), & x_{2}+x_{3} \cos \left(q_{2}\right), \\
x_{2}+x_{3} \cos \left(q_{2}\right), & x_{2},
\end{array}\right], \\
C(q, \dot{q}) & =\left[\begin{array}{cc}
-x_{3} \dot{q}_{2} \sin \left(q_{2}\right), & -x_{3}\left(\dot{q}_{1}+\dot{q}_{2}\right) \sin \left(q_{2}\right), \\
x_{3} \dot{q}_{1} \sin \left(q_{2}\right), & 0,
\end{array}\right],
\end{aligned}
$$

$$
G(q)=\left[\begin{array}{c}
15 g \cos q_{1}+8.75 g \cos \left(q_{1}+q_{2}\right) \\
8.75 g \cos \left(q_{1}+q_{2}\right)
\end{array}\right],
$$

where $x_{1}=13.33, x_{2}=8.98, x_{3}=8.75$, and $g=9.8$. The expected trajectories of the two-joint manipulators are $q_{1 d}=\sin (3 \pi t)$ and $q_{2 d}=\cos (3 \pi t)$, respectively. According to the above analysis, this paper uses three kinds of controllers to carry out simulation comparison analysis. Controller 1 is the controller designed in this paper, and the control input is expressed as

$$
\begin{aligned}
\tau= & M^{1-\alpha}\left(-\widehat{c}_{1}|s|^{b} \operatorname{sign}(s)-c_{2} \operatorname{arsh}(s)-k_{1} a_{1}|e|^{k_{1}-1} \dot{e}-\operatorname{sat}\left(k_{2} a_{2}|e|^{k_{2}-1} \dot{e}, h\right)\right)+C \dot{q}+G \\
& +M \ddot{q}_{d}-M c_{3} \operatorname{sign}(s)-M \widehat{d} .
\end{aligned}
$$

Controller 2 adopts the sliding mode surface designed by [22], which is expressed as follows:

$$
s=\dot{e}+a_{1}|e|^{k_{1}} \operatorname{sign}(e)+a_{2}|e|^{k_{2}} \operatorname{sign}(e) .
$$

The control input of the manipulator system can be obtained as

$$
\begin{aligned}
\tau= & M\left(-\widehat{c}_{1}|s|^{b} \operatorname{sign}(s)-c_{2} \operatorname{arsh}(s)-k_{1} a_{1}|e|^{k_{1}-1} \dot{e}-\operatorname{sat}\left(k_{2} a_{2}|e|^{k_{2}-1} \dot{e}, h\right)\right)+C \dot{q}+G \\
& +M \ddot{q}_{d}-M c_{3} \operatorname{sign}(s)-M \hat{d} .
\end{aligned}
$$

The difference between controller 2 and controller 1 designed in this paper is whether a fractional-order operator is introduced. This paper will verify the improvement of the tracking accuracy of the manipulator by the introduction of the fractional order through subsequent simulation experiments. For controller 3, the fractional-order sliding mode surface designed in this paper is as follows:

$$
s=D^{\alpha} e+a_{1}|e|^{o_{1}} \operatorname{sign}(e)+a_{2}|e|^{o_{2}} \operatorname{sign}(e) .
$$

In the same way, combining the adaptive VEPRL and the disturbance observer designed in this paper, the control input of the manipulator can be obtained as

$$
\begin{aligned}
\tau= & M^{1-\alpha}\left(-\widehat{c}_{1}|s|^{b} \operatorname{sign}(s)-c_{2} \operatorname{arsh}(s)-o_{1} a_{1}|e|^{o_{1}-1} \dot{e}-\operatorname{sat}\left(o_{2} a_{2}|e|^{o_{2}-1} \dot{e}, h\right)\right)+C \dot{q}+G \\
& +M \ddot{q}_{d}-M c_{3} \operatorname{sign}(s)-M \widehat{d} .
\end{aligned}
$$

The difference between controller 3 and controller 1 is that, for controller 3 , the last two exponential coefficients of the fractional sliding mode surface are fixed values. The value of the index term of the fractional sliding mode surface corresponding to controller 1 can be adjusted adaptively according to the size of the system state, which further accelerates the convergence speed. In order to ensure fairness and rationality in the analysis process, the initial position of the manipulator system is $\left[q_{1}, \dot{q}_{1}, q_{2}, \dot{q}_{2}\right]^{T}=[2,0,3,0]^{T}$, the three controller parameters are the same, and the sliding mode surface parameters are $\alpha=0.8, a_{1}=a_{2}=0.5, o_{1}=9 / 5$, and $o_{2}=5 / 9$, respectively. The parameters of the adaptive VEPRL are $c_{\operatorname{lmax}}=7$, $p=40, p_{1}=0.001, c_{2}=6$, and $c_{3}=0.001$. The disturbance observer parameters are $\lambda=25, \quad \nu_{1}=14, \nu_{2}=61$, $\kappa=0.6$, and $\xi=1.2$, respectively. The unknown disturbance is unified as $\tau_{d}=0.2 \sin (\pi t)$. The simulation results are shown in Figures 1-5.

It can be seen from Figure 1 that all three controllers can track the desired trajectory quickly and accurately, which proves that the introduction of the saturation function does not affect the accuracy of the manipulator control system too much. Compared with the other two controllers, controller 1 designed in this paper converges to the desired trajectory the fastest. The comparison between controller 1 and controller 2 proves that the introduction of fractional order effectively improves the convergence speed of the system state and speeds up the response speed of the system. Secondly, by comparing controller 1 and controller 3 , the analysis shows that, by adaptively adjusting the exponential coefficient of 


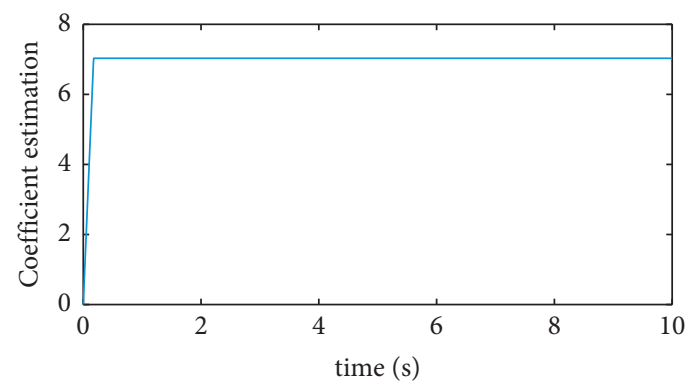

— Controller 1

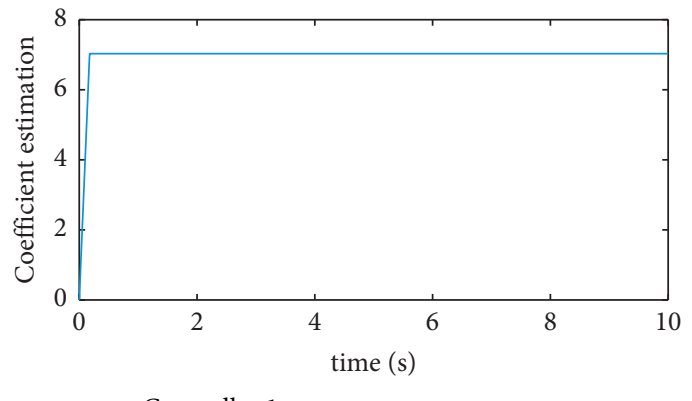

— Controller 1

FIgURE 4: Reaching law coefficient $c_{1}$ estimation.

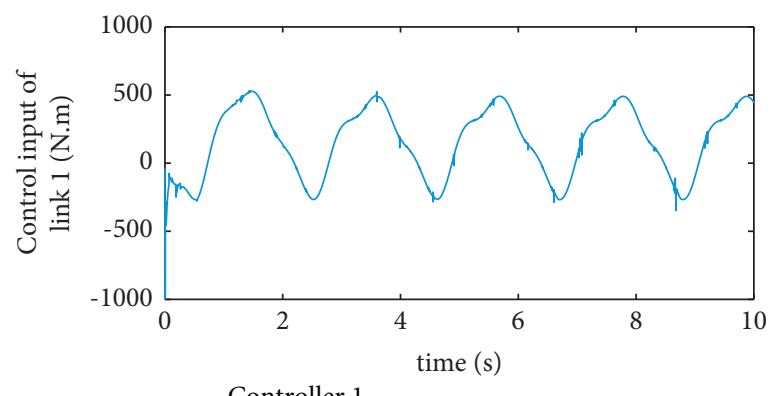

- Controller 1

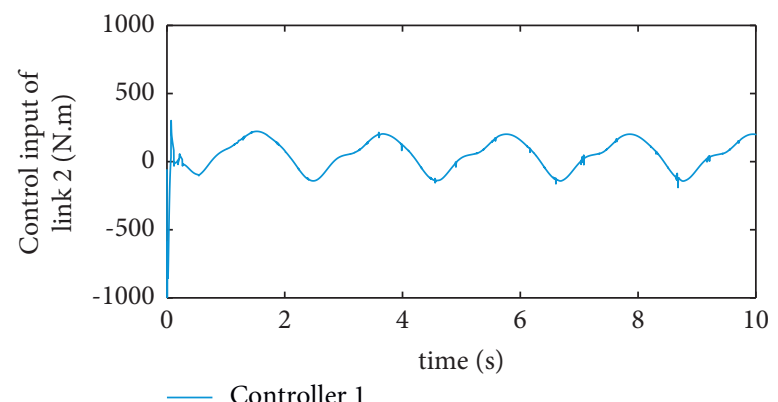

Figure 5: Control input.

the fractional-order sliding mode surface, the value of the exponential term can be adaptively changed according to the size of the system state, which speeds up the system speed at which the state approaches the sliding mode surface. It can be obtained from Figure 2 that, under the action of controller 1 designed in this paper, the system state converges to the equilibrium point the fastest. In order to quantitatively analyze the control accuracy of the three controllers, this paper introduces the root mean square error (RMSE) value to analyze the tracking error. This paper calculates the RMSE from $0 \mathrm{~s}$ to $3 \mathrm{~s}$ after stabilization, as shown in Tables 1 and 2 .

It can be seen more clearly from Tables 1 and 2 that controller 1 designed in this paper has the best tracking accuracy and tracking speed. It further illustrates that the introduction of fractional order can not only improve the convergence speed of the system state but also improve the 

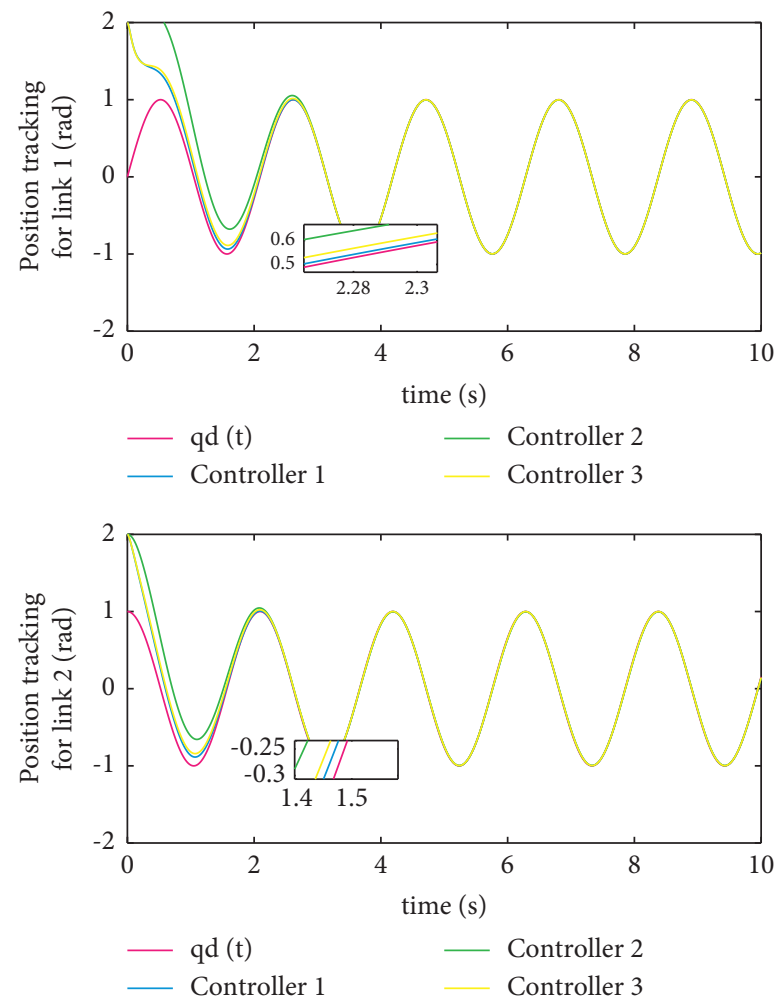

Figure 6: Comparison of manipulator trajectory tracking.
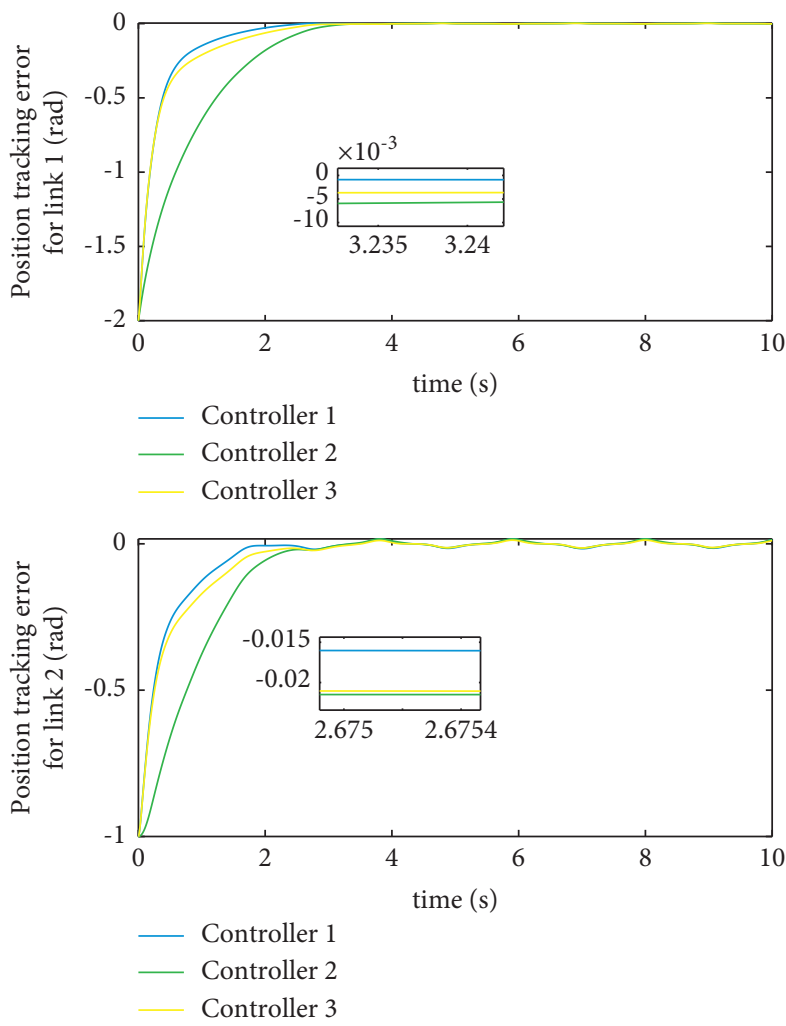

FIgURE 7: Comparison of tracking error of the manipulator. 

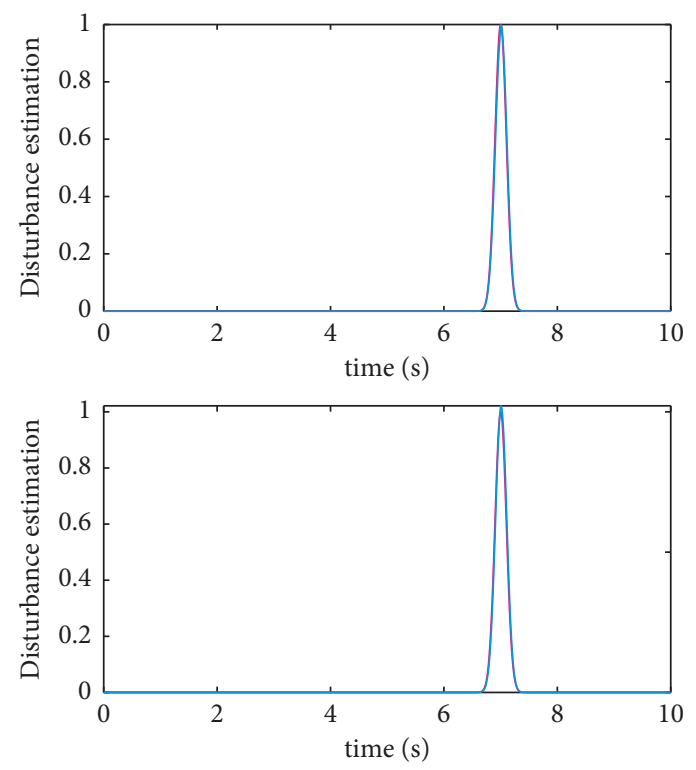

FIgURE 8: Disturbance estimation.

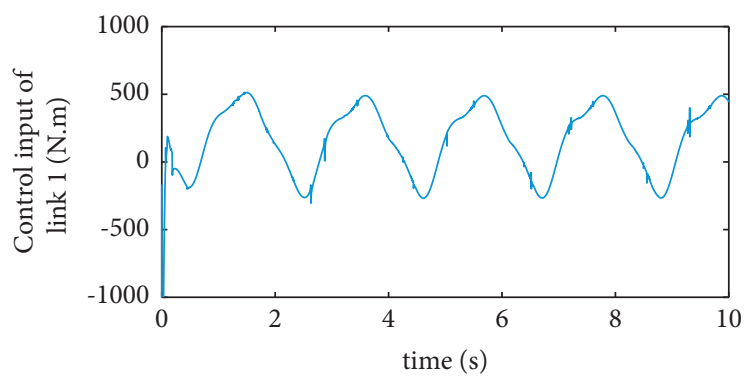

Controller 1

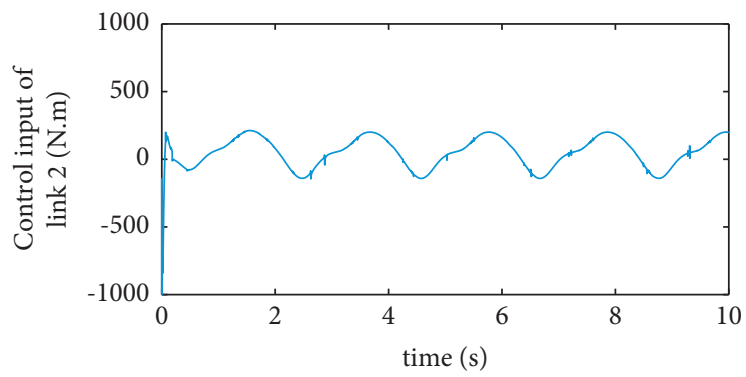

Controller 1

Figure 9: Control input.

tracking accuracy of the manipulator control system. It can be seen from Figure 3 that the disturbance observer introduced in this paper can effectively estimate the magnitude of the unknown disturbance from the outside world, thereby performing feedforward compensation for the control system and effectively improving the accuracy of the control system. Figure 4 shows the estimated value of the reaching law coefficients, through the adaptive adjustment of the reaching law coefficients, and the robustness of the manipulator control system is enhanced. Figure 5 is the control input of controller 1 designed in this paper. It can be seen from Figure 5 that the controller designed in this paper has only a small amplitude chattering phenomenon, which is suitable for the actual situation. In summary, the controller designed in this paper has strong robustness, high tracking accuracy, and fast dynamic response, which effectively improves the trajectory tracking accuracy and speed of the manipulator system.

Considering that the manipulator system is susceptible to different types of unknown disturbance. In order to reflect the superiority of the controller designed in this paper, set $\tau_{\mathrm{d}}=1 \exp \left(-(t-7)^{2} /\left(2 \times 0.1^{2}\right)\right)$ and perform simulation 
comparison again and change the initial position of the manipulator system to $\left[q_{1}, \dot{q}_{1}, q_{2}, \dot{q}_{2}\right]^{T}=[2,0,2,0]^{T}$; the rest of the controller parameters are unchanged. The simulation results are shown in Figures 6-9.

Similar to the previous analysis, a brief description is used. It can be seen from Figures 6 and 7 that, under the action of controller 1 designed in this paper, compared with the other two controllers, the system state has certain advantages in terms of convergence speed and tracking accuracy. It can be seen from Figure 8 that the disturbance observer introduced in this paper can quickly and accurately estimate the sudden disturbance $\tau_{d}$, so as to perform feedforward compensation for the manipulator control system and improve the tracking accuracy of the system. It can be seen from Figure 9 that the system chattering amplitude is relatively small.

\section{Conclusion}

Aiming at the trajectory tracking problem of the manipulator, this paper proposes an adaptive FONFTSM controller. The controller is divided into three parts. First, the fractional-order sliding mode surface and adaptive VEPRL are designed in the reaching mode and sliding mode, respectively, and effectively improve the convergence speed and tracking accuracy of the system state in the whole process. By introducing a disturbance observer to estimate the unknown external disturbance in real time, chattering is reduced, and the accuracy of the manipulator control system is improved. Finally, the stability of the control system is proved by the Lyapunov function. Comparison with different controllers through simulation experiments proves that the introduction of fractional-order operators can effectively improve the accuracy and convergence speed of the control system. At the same time, the controller designed in this paper has strong robustness and better tracking accuracy. The controller designed in this paper is also suitable for other nonlinear systems. The next step will be to verify the effectiveness of the controller designed in this paper in the actual system. At the same time, consider the problem of control input saturation and balance the relationship between the convergence speed of the system state and the control input.

\section{Data Availability}

The data used to support the findings of this study are available from the corresponding author upon request.

\section{Conflicts of Interest}

The authors declare no potential conflicts of interest with respect to the research, authorship, and/or publication of this article.

\section{Acknowledgments}

This work was supported by the Natural Science Foundation of Gansu Province (20JR5RA419) and Lanzhou Jiaotong University-Tianjin University Innovation Fund Project (2019053).

\section{References}

[1] C. Li, X. Gu, and X. Xiao, "A robotic system with multichannel flexible parallel manipulators for single port access surgery," IEEE Transactions on Industrial Informatics, vol. 15, pp. 1678-1687, 2018.

[2] X. Liang, H. Cheong, and Y. Sun, "Design, characterization, and implementation of a two-DOF fabric-based soft robotic arm," IEEE Robotics and Automation Letters, vol. 3, pp. 2702-2709, 2018.

[3] P. R. Soria, B. C. Arrue, and A. Ollero, "A 3d-printable docking system for aerial robots: controlling aerial robotic manipulators in outdoor industrial applications," IEEE Robotics and Automation Magazine, vol. 26, pp. 44-53, 2019.

[4] C. Yang, H. Wu, and Z. Li, "Mind control of a robotic arm with visual fusion technology," IEEE Transactions on Industrial Informatics, vol. 14, pp. 3822-3830, 2017.

[5] Y. Sun, J. Xu, and H. Wu, "Deep learning based semi-supervised control for vertical security of maglev vehicle with guaranteed bounded airgap," IEEE Transactions on Intelligent Transportation Systems, vol. 22, pp. 4431-4442, 2021.

[6] S. Han, H. Wang, and Y. Tian, "Model-free based adaptive nonsingular fast terminal sliding mode control with timedelay estimation for a 12 DOF multi-functional lower limb exoskeleton," Advances in Engineering Software, vol. 119, pp. 38-47, 2018.

[7] T. Song, L. Fang, and H. Wang, "Model-free finite-time terminal sliding mode control with a novel adaptive sliding mode observer of uncertain robot systems," Asian Journal of Control, vol. 32, 2021.

[8] Y. Sun, J. Xu, and G. Lin, "RBF neural network-based supervisor control for maglev vehicles on an elastic track with network time-delay," IEEE Transactions on Industrial Informatics, vol. 54, 2020.

[9] Y. Wang, L. Gu, and Y. Xu, "Practical tracking control of robot manipulators with continuous fractional-order nonsingular terminal sliding mode," IEEE Transactions on Industrial Electronics, vol. 63, pp. 6194-6204, 2016.

[10] Y. Wang, F. Yan, and S. Jiang, "Time delay control of cabledriven manipulators with adaptive fractional-order nonsingular terminal sliding mode," Advances in Engineering Software, vol. 121, pp. 13-25, 2018.

[11] J. Baek, M. Jin, and S. Han, "A new adaptive sliding-mode control scheme for application to robot manipulators," IEEE Transactions on Industrial Electronics, vol. 63, no. 6, pp. 3628-3637, 2016.

[12] Z. Kuang, H. Gao, and M. Tomizuka, "Precise linear-motor synchronization control via cross-coupled second-order discrete-time fractional-order sliding mode," IEEE, vol. 26, pp. 358-368, 2020.

[13] Z. Ma, Z. Liu, and P. Huang, "Fractional-order control for uncertain teleoperated cyber-physical system with actuator fault," IEEE, vol. 43, 2020.

[14] D. Nojavanzadeh and M. Badamchizadeh, "Adaptive fractional-order non-singular fast terminal sliding mode control for robot manipulators," IET Control Theory \& Applications, vol. 10, pp. 1565-1572, 2016.

[15] Y. Wang, F. Yan, and K. Zhu, "A new practical robust control of cable-driven manipulators using time-delay estimation," International Journal of Robust and Nonlinear Control, vol. 29, pp. 3405-3425, 2019.

[16] X. Yin, L. Pan, and S. Cai, "Robust adaptive fuzzy sliding mode trajectory tracking control for serial robotic manipulators," 
Robotics and Computer-Integrated Manufacturing, vol. 72, Article ID 101884, 2021.

[17] K. Zheng, Y. Hu, and B. Wu, "Intelligent fuzzy sliding mode control for complex robot system with disturbances," European Journal of Control, vol. 51, pp. 95-109, 2020.

[18] M. Vijay and D. Jena, "Backstepping terminal sliding mode control of robot manipulator using radial basis functional neural networks," Computers \& Electrical Engineering, vol. 67, pp. 690-707, 2018.

[19] Y. Tang, "Terminal sliding mode control for rigid robots," Automatica, vol. 34, pp. 51-56, 1998.

[20] L. Yang and J. Yang, "Nonsingular fast terminal sliding-mode control for nonlinear dynamical systems," International Journal of Robust and Nonlinear Control, vol. 21, pp. 18651879, 2011.

[21] Y. Feng, X. Yu, and Z. Man, "Non-singular terminal sliding mode control of rigid manipulators," Automatica, vol. 38, no. 12, pp. 2159-2167, 2002.

[22] Y. Tian, Y. Cai, and Y. Deng, “A fast nonsingular terminal sliding mode control method for nonlinear systems with fixed-time stability guarantees," IEEE Access, vol. 8, pp. 60444-60454, 2020.

[23] W. Jie, Z. Yudong, and B. Yulong, "Trajectory tracking control using fractional-order terminal sliding mode control with sliding perturbation observer for a 7-DOF robot manipulator," IEEE, vol. 25, pp. 1886-1893, 2020.

[24] T. M. Duc, N. Van Hoa, and T.-P. Dao, “Adaptive fuzzy fractional-order nonsingular terminal sliding mode control for a class of second-order nonlinear systems," Journal of Computational and Nonlinear Dynamics, vol. 13, 2018.

[25] A. K. Junejo, W. Xu, and C. Mu, "Adaptive speed control of PMSM drive system based a new sliding-mode reaching law," IEEE Transactions on Power Electronics, vol. 35, pp. 1211012121, 2020.

[26] S. M. Mozayan, M. Saad, and H. Vahedi, "Sliding mode control of PMSG wind turbine based on enhanced exponential reaching law," IEEE Transactions on Industrial Electronics, vol. 63, pp. 6148-6159, 2016.

[27] Y. Zhang, X. Yang, and P. Wei, "Fractional-order adaptive non-singular fast terminal sliding mode control with time delay estimation for robotic manipulators," IET Control Theory \& Applications, vol. 14, pp. 2556-2565, 2020.

[28] Y. Kali, M. Saad, and K. Benjelloun, "Super-twisting algorithm with time delay estimation for uncertain robot manipulators," Nonlinear Dynamics, vol. 93, pp. 557-569, 2018.

[29] Y. Wang, J. Chen, and F. Yan, "Adaptive super-twisting fractional-order nonsingular terminal sliding mode control of cable-driven manipulators," ISA Transactions, vol. 86, pp. 163-180, 2019.

[30] P. Zhu, Y. Chen, and M. Li, "Fractional-order sliding mode position tracking control for servo system with disturbance," ISA Transactions, vol. 105, pp. 269-277, 2020.

[31] W. Weibing Gao and J. C. Hung, "Variable structure control of nonlinear systems: a new approach," IEEE Transactions on Industrial Electronics, vol. 40, no. 1, pp. 45-55, 1993.

[32] H. Li and Y. Cai, "Sliding mode control with double power reaching law," Control and Decision, vol. 31, pp. 498-502, 2016.

[33] J. Pan, W. Li, and H. Zhang, "Control algorithms of magnetic suspension systems based on the improved double exponential reaching law of sliding mode control," International Journal of Control, Automation and Systems, vol. 16, pp. 2878-2887, 2018.
[34] G.-Y. Yang and S.-Y. Chen, "Piecewise fast multi-power reaching law: basis for sliding mode control algorithm," Measurement and Control, vol. 53, pp. 1929-1942, 2020.

[35] J. Li, H. Du, and Y. Cheng, "Position tracking control for permanent magnet linear motor via fast nonsingular terminal sliding mode control," Nonlinear Dynamics, vol. 97, pp. 2595-2605, 2019.

[36] Y. Zhu and J. Fei, "Disturbance observer based fuzzy sliding mode control of PV grid connected inverter," IEEE Access, vol. 6, pp. 21202-21211, 2018.

[37] A. A. Kilbas, H. M. Srivastava, and J. J. Trujillo, Theory and Applications of Fractional Differential Equations, Elsevier, Amsterdam, The Netherlands, 2006.

[38] N. Aguila-Camacho, M. A. Duarte-Mermoud, and J. A. Gallegos, "Lyapunov functions for fractional order systems," Communications in Nonlinear Science and $\mathrm{Nu}$ merical Simulation, vol. 19, no. 9, pp. 2951-2957, 2014.

[39] Z. Zhu, Y. Xia, and M. Fu, "Attitude stabilization of rigid spacecraft with finite-time convergence," International Journal of Robust and Nonlinear Control, vol. 21, pp. 686-702, 2011.

[40] R. Wu, C. Wei, and F. Yang, "FxTDO -based non-singular terminal sliding mode control for second-order uncertain systems," IET Control Theory \& Applications, vol. 12, pp. 2459-2467, 2018.

[41] Y. Feng, X. Yu, and F. Han, "On nonsingular terminal slidingmode control of nonlinear systems," Automatica, vol. 49, no. 6, pp. 1715-1722, 2013. 